\title{
Bedside diagnosis of pleural effusion with a latest generation hand-carried ultrasound device in intensive care patients
}

\author{
Stephan Schleder', C Dornia ${ }^{1}$, F Poschenrieder ${ }^{1}$, L DendI ${ }^{1}$, L Cojocaru², T Bein ${ }^{3}$, C Schmid $^{4}$, \\ C Stroszczynski ${ }^{1}$, EM Jung ${ }^{1}$ and $P$ Heiss ${ }^{1}$ \\ ${ }^{1}$ Department of Radiology; ${ }^{2}$ Department of Nuclear Medicine; ${ }^{3}$ Department of Surgery; ${ }^{4}$ Department of Cardiothoracic Surgery, University \\ Medical Center Regensburg, Regensburg, Germany \\ Correspondence to: Stephan Schleder. Email: Stephan.schleder@ukr.de
}

\begin{abstract}
Background: Further development established hand-carried ultrasound (HCU) imagers in daily clinical workflow providing several advantages such as fast bedside availability and prompt diagnosis.

Purpose: To evaluate the diagnostic yield of a latest generation $\mathrm{HCU}$ imager compared to chest radiography (CR) for the detection of pleural effusion (PE) in intensive care patients.

Material and Methods: Forty-eight hemithoraces of 24 patients on surgical intensive care units were enrolled in this study. All hemithoraces were evaluated using both HCU and CR. Definite diagnosis of PE was achieved using a high-end ultrasound system as standard of reference. Statistical analysis was performed using $2 \times 2$ tables and a McNemar test. A $P$ value of $<0.05$ was considered statistically significant.

Results: PE was present in 35 of 48 hemithoraces $(73 \%)$. The HCU examination was carried out technically successfully in all hemithoraces. Sensitivity and specificity of HCU for the diagnosis of PE was $91 \%$ and $100 \%$, respectively, whereas sensitivity and specificity of CR was $74 \%$ and $31 \%$, respectively. The difference between $\mathrm{HCU}$ and $\mathrm{CR}$ was statistically significant with respect to specificity but not sensitivity $(P=0.008$ and $P=0.11$, respectively).

Conclusion: Due to its ease of use and its high diagnostic yield HCU systems of the latest generation constitute a helpful technique for the primary assessment of PE.
\end{abstract}

Keywords: Ultrasound, pleura, conventional radiography, thorax

Submitted December 9, 2011; accepted for publication March 4, 2012

In patients being treated in intensive care units pleural effusion (PE) is a common complication (1). Usually the diagnosis of pleural effusion is based on physical examination and chest radiography. However, the diagnostic accuracy of physical examination is low, even when performed by experienced operators (2). Furthermore chest radiography may underestimate the amount of pleural effusion, in particular when the image is taken in a sitting or supine position. A minimum of $200 \mathrm{~mL}$ of pleural fluid is necessary to detect costophrenic obliteration in posteroanterior radiographs, but up to $500 \mathrm{~mL}$ of pleural fluid can be present without detectable costophrenic obliteration in some cases (3). In the supine position more than $500 \mathrm{~mL}$ of pleural fluid must be present to be detectable in chest radiographs (4).

Chest ultrasound is a useful and non-invasive tool for the evaluation of PE as shown by prior studies (5).
Ultrasound can even reveal very small amounts of pleural fluid, as little as $3-5 \mathrm{~mL}$ (6). Additionally thoracentesis can be performed under ultrasound guidance $(7,8)$. Further advantages of chest ultrasound are low costs, lack of radiation exposure, and userfriendliness.

Further development generated miniaturized ultrasound systems leading to an introduction of hand-carried ultrasound (HCU) imagers in daily clinical workflow. The major advantage of these devices is a fast bedside availability allowing prompt diagnosis and immediate therapeutic measures (9).

In the present study we evaluated the diagnostic yield of a latest generation $\mathrm{HCU}$ imager in comparison to chest radiography for the recognition of $\mathrm{PE}$ in intensive care patients. 


\section{Material and Methods}

\section{Patients}

On two consecutive days all 24 patients (48 hemithoraces) on a cardiothoracic and general surgical intensive care unit for whom a supine chest radiograph was ordered by the ward physician were additionally examined with both hand-carried and high-end ultrasound. Patients were enrolled in this study irrespective of their underlying disease. There was no exclusion criterion. The study operators (SS, CD, FP) who carried out the ultrasound examination and the reading of the chest radiographs were unaware of the indication for the supine chest radiograph and the patients' underlying disease. The patients' basic demographic data are shown in Table 1. Written informed consent was obtained from all patients and the study was approved by the institutional ethics committee.

\section{Chest radiography}

Indications for supine chest radiography were clinical signs of cardiac decompensation, clinically suspected pneumonia, PE or atelectasis and the exclusion of a pneumothorax after central vein catheterization in six, five, five, four, and four patients, respectively. All radiographs were acquired on the intensive care units using a mobile $\mathrm{X}$-ray device (Mobilett XP ECO, Siemens Healthcare, Erlangen, Germany). All chest radiographs were evaluated for the presence of PE by the same radiologist (FP), who had specialized in cardiopulmonary imaging for 4 years and who was unaware of the ultrasound results. All chest radiographs were included and each hemithorax was scored separately.

\section{Chest examination with hand-carried ultrasound}

The HCU examination was carried out using a latest generation device (VScan, GE Healthcare, Waukesha, WI, USA)

Table 1 Basic demographic characteristics of all enrolled patients including frequency and type of surgery

\begin{tabular}{|c|c|c|}
\hline & $n$ & $\%$ \\
\hline Patients & 24 & 100 \\
\hline Hemithoraces & 48 & 100 \\
\hline \multicolumn{3}{|l|}{ Gender } \\
\hline Male & 14 & 58 \\
\hline Female & 10 & 42 \\
\hline Median age and age range (years) & $65(42-91)$ & \\
\hline Median weight and weight range (kg) & $86(58-122)$ & \\
\hline $\begin{array}{l}\text { Patients without prior surgery/no surgery } \\
\text { planned }\end{array}$ & 4 & 17 \\
\hline Patients with prior surgery & 20 & 83 \\
\hline $\begin{array}{l}\text { Median time interval and time range between } \\
\text { surgery and examination (days) }\end{array}$ & $3(1-6)$ & \\
\hline \multicolumn{3}{|l|}{ Type and frequency of surgery } \\
\hline Coronary bypass & 4 & 20 \\
\hline Lung lobectomy & 4 & 20 \\
\hline Cardiac valve prosthesis & 3 & 15 \\
\hline Hemicolectomy & 2 & 10 \\
\hline Pleurodesis & 2 & 10 \\
\hline Whipple's pancreaticoduodenectomy & 2 & 10 \\
\hline Major trauma & 2 & 10 \\
\hline Liver transplantation & 1 & 5 \\
\hline
\end{tabular}

with a plane 1.7-3.8 MHz transducer for two-dimensional imaging and a 3.5-inch color LCD-display The probe measures $120 \times 33 \times 26 \mathrm{~mm}$. The weight of this device is $390 \mathrm{~g}$. The total scan time is approximately $62 \mathrm{~min}$ with fully-charged batteries. A picture of the HCU device is shown in Fig. 1. The chest ultrasound examination with the HCU imager was performed within a maximum of $3 \mathrm{~h}$ after the acquisition of supine chest radiograph. The operator (SS) was unaware of the results of both chest radiography and high-end ultrasound.

\section{Chest examination with high-end ultrasound}

Chest examination with the high-end ultrasound system (LOGIQ E9, GE Healthcare, Waukesha, WI, USA) with a curved-array 1-5 MHz transducer was carried out as standard of reference for the assessment of pleural effusion. The operator (CD) was unaware of the findings of both chest radiography and HCU. The chest ultrasonography with the high-end ultrasound system was performed within a maximum of $15 \mathrm{~min}$ after the HCU examination.

\section{Technique of ultrasound}

In order to standardize and simplify the ultrasound examinations all patients were examined lying in their beds in intensive care units. In particular, none of the patients was moved for the examination with the HCU or high-end ultrasound system. Both hemithoraces were examined without modifications of the patients' breathing frequency. The intercostal spaces were used us ultrasound windows. In all hemithoraces at least two intercostal ultrasound windows were employed to scan the dorsal and lateral aspects of the basal pleural space for the presence of PE. A PE was diagnosed when an anechoic space between the parietal pleura and the highly reflective visceral pleura-lung interface was present (9). An echoic space between the parietal pleura and the highly reflective visceral pleura-lung interface was considered to represent severe complications such as empyema or hemothorax. Neither thoracentesis nor other interventions that could have influenced the amount of pleural effusion were performed between the acquisition of the chest radiograph and the acquisition of the HCU and high-end ultrasound examination.

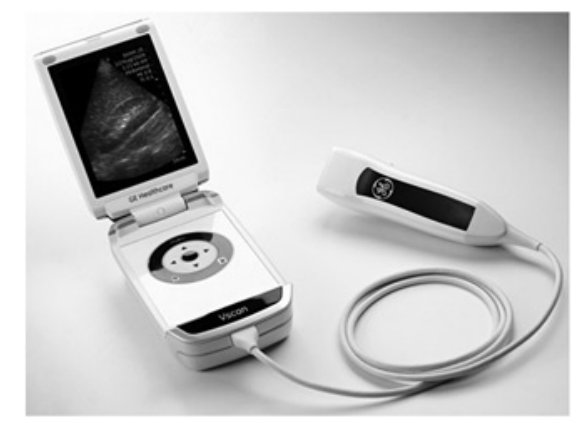

Fig. 1 Image of the study hand-carried ultrasound device (VScan, GE Healthcare, Waukesha WI, USA) with kind permission of Mr Bastian Werminghoff (GE Healthcare Germany, Munich, Germany) 
Table 2 Sensitivity, specificity, positive and negative predictive value for the diagnosis of pleural effusion using hand-carried ultrasound and chest radiography with high-end ultrasound as standard of reference

\begin{tabular}{lcll}
\hline & $\begin{array}{l}\text { Hand-carried } \\
\text { ultrasound }\end{array}$ & $\begin{array}{l}\text { Chest } \\
\text { radiography }\end{array}$ & $\begin{array}{l}\boldsymbol{P} \\
\text { value }\end{array}$ \\
\hline Sensitivity & $91 \%$ & $74 \%$ & 0.11 \\
$\begin{array}{l}\text { Specificity } \\
\begin{array}{l}\text { Positive predictive } \\
\text { value }\end{array}\end{array}$ & $100 \%$ & $31 \%$ & 0.008 \\
$\begin{array}{l}\text { Negative } \\
\text { predictive value }\end{array}$ & $82 \%$ & $73 \%$ & \\
\hline
\end{tabular}

\section{Statistical analysis}

Data were collected using Excel tables (Excel 2007, Microsoft, Redmond, WA, USA). Statistical analysis was performed using PASW 18 (PASW v. 18, IBM SPSS, Inc., Armonk, NY, USA). Agreement between the findings of hand-carried ultrasound or chest radiography and the definite diagnosis generated by high-end ultrasound was assessed from $2 \times 2$ tables. Comparison of sensitivity and specificity between hand-carried ultrasound and chest radiography was made using the McNemar test. A $P$ value of $<0.05$ was considered statistically significant.

\section{Results}

Chest ultrasound with the high-end ultrasound system, the standard of reference, depicted PE in 21 of 24 patients $(87.5 \%)$ and in 35 of 48 hemithoraces $(73 \%)$. Bilateral PE was found in 14 of 24 patients (58\%), whereas unilateral pleural fluid on the right or left side was present in three patients $(13 \%)$ and four patients $(17 \%)$, respectively.

HCU was carried out technically successfully in all 24 patients and in all 48 hemithoraces. In the operator's experience the employed HCU system exhibited convenient handling, including: (a) quick availability with a boot time of less than $20 \mathrm{~s}$; (b) comfortable scanning of the hemithoraces of the lying patients with the small plane transducer; (c) clear reading of the images on the 3.5-inch color LCD display; as well as (d) easy storage of patient data and ultrasound images. Mean scanning time of the HCU examination was $1.5 \mathrm{~min}$ with a range of 1-3 min. Examination with HCU revealed pleural effusion correctly in 32 out of 35 hemithoraces and showed no false-positive results. Thus, sensitivity and specificity were $91 \%$ and $100 \%$, respectively. In the remaining three cases where HCU examination showed false-negative results the amount of PE that was identified by the high-end ultrasound system was estimated to be less than $50 \mathrm{~mL}$. The weight of the three patients with false-negative findings (96, 115, and $122 \mathrm{~kg}$, respectively) was in the upper range of all 24 patients (median weight (a)

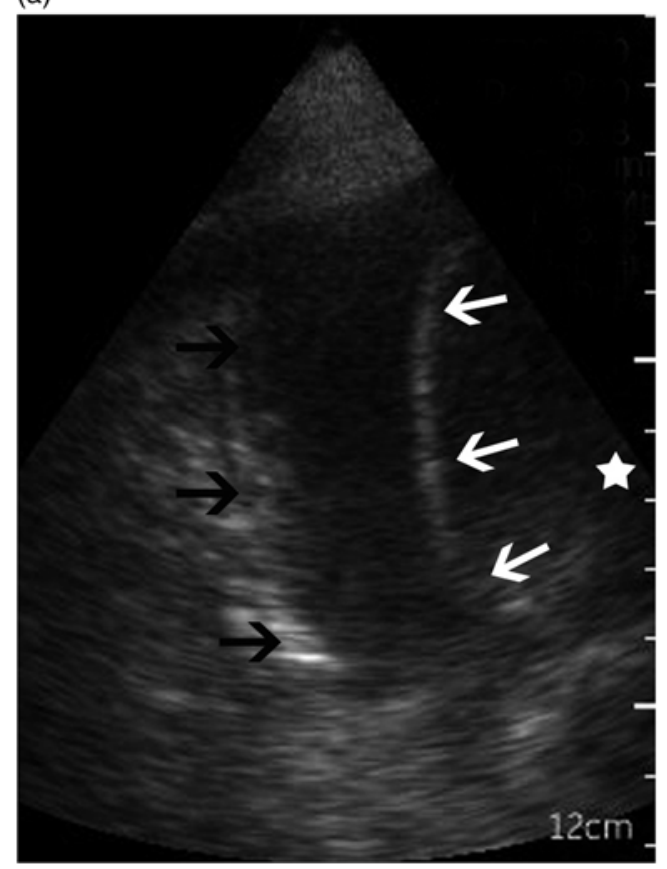

(b)

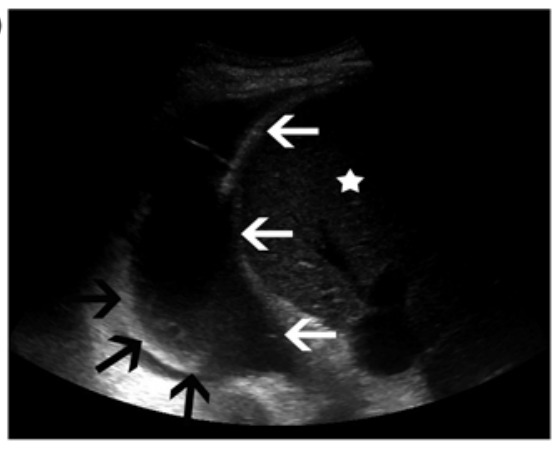

(c)

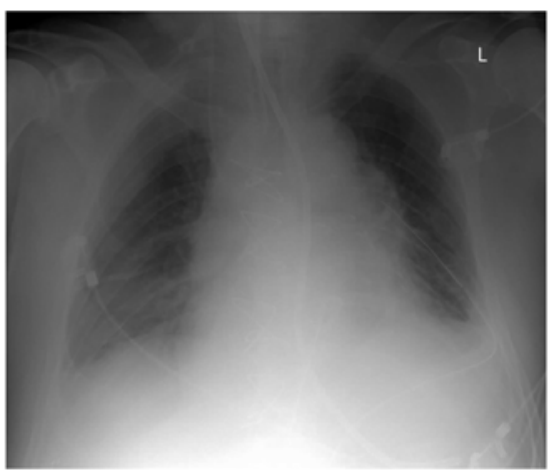

Fig. 2 Incongruent findings of hand-carried ultrasound and chest radiograph in an 83-year-old male patient for whom a chest radiograph was carried out for the diagnostic workup of pneumonia 4 days after coronary bypass surgery. High-end ultrasound as well as hand-carried ultrasound depicted a right-sided pleural effusion, whereas chest radiograph was false-negative for the diagnosis of pleural effusion in this patient. (a) Hand-carried ultrasound B-Scan depicts right-sided pleural effusion which is characterized as the anechoic space between the highly reflective visceral pleura (black arrows) and the parietal pleura (white arrows). To note, the higher spatial resolution of the high-end ultrasound (b) compared with the hand-carried ultrasound system (a) is clearly visible (e.g. of the liver parenchyma, marked with a white star). (b) B-Scan with high-end ultrasound system exhibits right-sided pleural effusion which is characterized as the anechoic space between the highly reflective visceral pleura (black arrows) and the parietal pleura (white arrows). To note, the higher spatial resolution of the high-end ultrasound (b) compared with the hand-carried ultrasound system (a) is clearly visible (e.g. of the liver parenchyma, marked with a white star). (c) Supine chest radiograph did not exhibit typical findings of pleural effusion, hence, in this patient absence of pleural effusion was diagnosed 
$86 \mathrm{~kg})$. Positive and negative predictive value for the diagnosis of PE with HCU was $100 \%$ and $82 \%$, respectively.

Chest radiography diagnosed PE correctly in 26 of 35 hemithoraces and was false-positive in nine out of 48 hemithoraces. Hence, sensitivity, specificity, positive and negative predictive value for the diagnosis of PE with chest radiography were $74 \%, 31 \%, 73 \%$, and $32 \%$, respectively.

Sensitivity of HCU (91\%) was superior to sensitivity of chest radiography $(74 \%)$ but the difference was statistically not significant $(P=0.11)$. Specificity of HCU $(100 \%)$ was higher than the specificity of chest radiography $(31 \%)$ and the difference was statistically significant $(P=0.008)$.

The results of sensitivity and specificity of HCU and chest radiography are summarized in Table 2 .

Figs. 2 and 3 demonstrate examples of incongruent and congruent findings of $\mathrm{HCU}$ and chest radiography in patients with PE.

\section{Discussion}

The present study shows that modern HCU imagers provide a very convenient, fast, and reliable technique for the diagnosis of $\mathrm{PE}$ in patients in intensive care units. In comparison to the standard of reference high-end ultrasound, HCU revealed no false-positive result and was false-negative in only three out of 35 hemithoraces leading to a sensitivity and specificity of $91 \%$ and $100 \%$, respectively. To note, the three patients with false-negative results were overweight which is associated with reduced image quality of $\mathrm{HCU}$ and, the operator with the high-end ultrasound system estimated the amount of pleural fluid to less than $50 \mathrm{~mL}$ (which is usually not of clinical significance). In addition, in our study population the diagnostic yield of HCU was superior to chest radiography.

In the present survey all ultrasound examinations were performed in the supine position and, in particular, the patients were not moved, e.g. into an upright position. This proceeding was chosen to enable convenient and fast ultrasonographic examination of the patients using $\mathrm{HCU}$ imagers and to prove that HCU reliably depicts PE in patients lying in this supine position.

Diverse HCU imagers have been tested in prior studies, e.g. the Acuson P10 (Siemens Healthcare, Erlangen, Germany) and the OptiGo, (Philips Medical Systems, Andover, CA, USA) (9), but to the best of our knowledge these are the first data on the diagnostic value of a latest generation HCU device for the assessment of pleural effusion in patients in intensive care units being examined lying in the supine position.

Former evaluated HCU imagers already showed promising results for the diagnosis of $\mathrm{PE}$, but these imagers have not made their way into daily clinical workflow yet. Instead, chest radiography remains the standard noninvasive diagnostic tool for the diagnosis of PE, probably related to the fact, that chest radiography is helpful to assess cardiopulmonary complications beyond PE like pneumonia, pulmonary atelectasis, and cardiac decompensation.

Considering the advantages of the HCU system employed in this study, including (a) ease and quickness
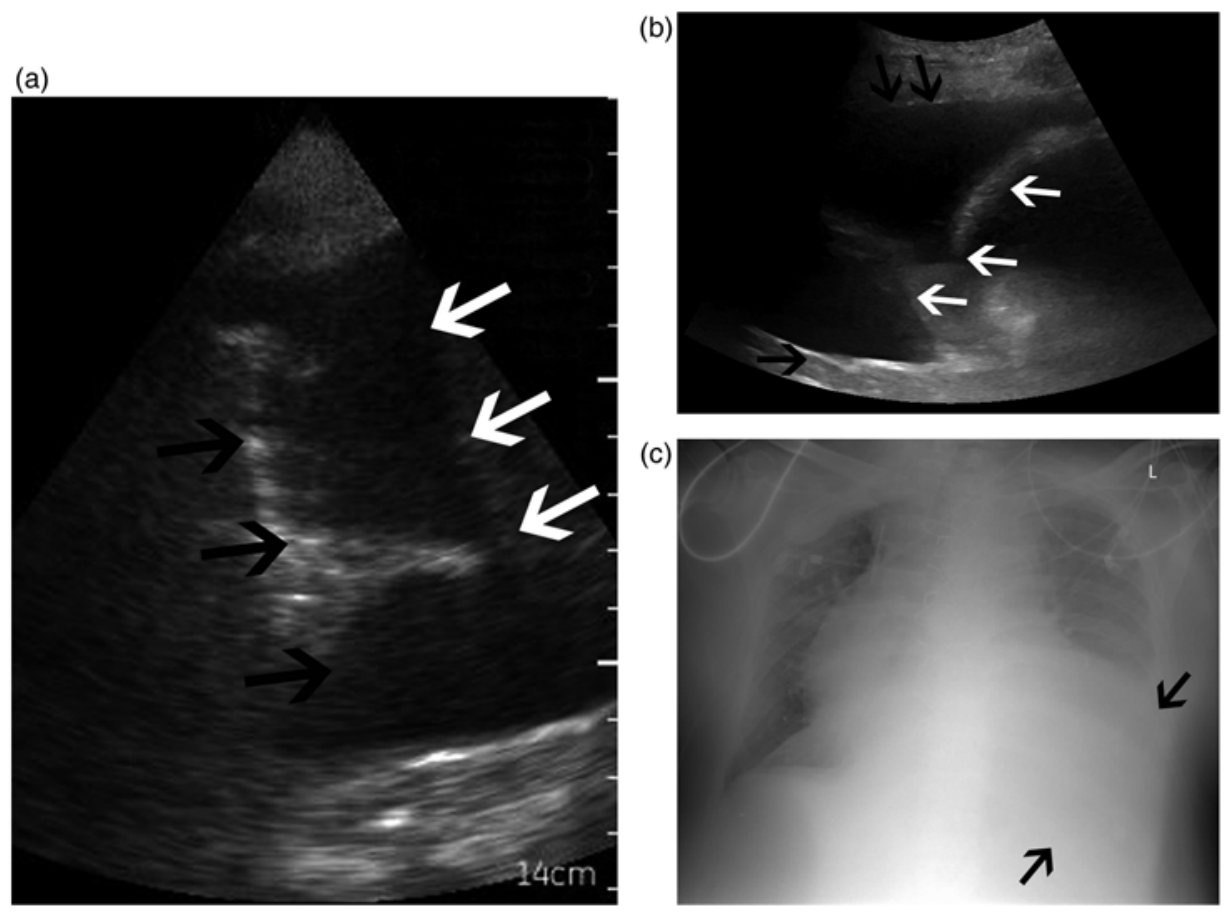

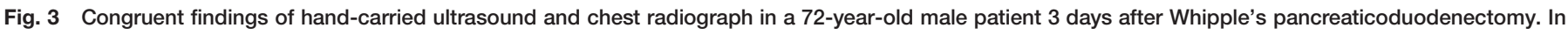
this patient a chest radiograph was performed in order to identify the cause for clinically diagnosed cardiac decompensation. Large left-sided pleural effusion was depicted by both the hand-carried and high-end ultrasound system as well as by chest radiography. (a) Hand-carried ultrasound B-Scan demonstrates left-sided pleural effusion which is characterized as the anechoic space between the highly reflective visceral pleura (black arrows) and the parietal pleura (white arrows). (b) High-end ultrasound system B-Scan exhibits left sided pleural effusion which is characterized as the anechoic space between the highly reflective visceral pleura

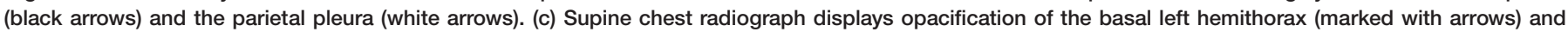
presence of pleural effusion to the left was diagnosed 
of use, (b) quick bedside availability, (c) lack of radiation exposure, (d) low costs, and (e) high diagnostic yield for the diagnosis of PE in patients lying in the supine position we recommend to primarily examine patients by HCU if $\mathrm{PE}$ is, for example, clinically a likely diagnosis. If HCU depicts significant amounts of pleural fluid prompt pleural drainage can be initiated. If consequently clinical symptoms disappear, no further investigations such as chest radiography are necessary. In patients in whom HCU was negative further investigation (e.g. by high-end ultrasound or chest radiography) is indicated, in particular when complications like pneumonia, pulmonary atelectasis, or cardiac decompensation are assumed $(10,11)$. In symptomatic patients with negative ultrasound and negative chest radiography, computed tomography of the thorax should be considered $(5,12,13)$.

There are some limitations of the present study. Only 24 patients and 48 hemithoraces were enrolled and examined with HCU, high-end ultrasound, and chest radiography by only one operator, respectively. Ultrasound is an operator-dependent modality and different operators might obtain different results. Nevertheless, in our study, a statistically significant difference of the specificity of HCU and chest radiography was determined. Computed tomography of the thorax is considered the "gold standard" for the diagnosis of PE (14). In the present study high-end ultrasound was chosen as standard of reference for the diagnosis of pleural effusion, because this approach is free of radiation exposure to the patients $(15,16)$ and, in a recently published survey Xirouchaki et al. showed that high-end ultrasound and computed tomography of the thorax reveal highly concordant results for the diagnosis of PE (17), revealing high-end ultrasound as a eligible standard of reference for the diagnosis of PE.

In conclusion, due to its ease of use and its high diagnostic yield HCU systems of the latest generation constitute a helpful technique for the primary assessment of PE in patients in intensive care units.
Conflict of interest: None.

\section{REFERENCES}

1 Peng MJ, Vargas FS, Cukier A, et al. Postoperative pleural changes after coronary revascularization. Comparison between saphenous vein and internal mammary artery grafting. Chest 1992;101:327-30

2 Diacon AH, Brutsche MH, Soler M. Accuracy of pleural puncture sites: a prospective comparison of clinical examination with ultrasound. Chest 2003;123:436-41

3 Colins JD, Burwell D, Furmanski S, et al. Minimal detectable pleural effusions. A roentgen pathology model. Radiology 1972;105:51-3

4 Woodring JH. Recognition of pleural effusion on supine radiographs: how much fluid is required? Am J Roentgenol 1984;142:59-64

5 Eibenberger KL, Dock WI, Ammann ME, et al. Quantification of pleural effusions: sonography versus radiography. Radiology 1994;191:681-4

6 Gryminski J, Krakowka P, Lypacewicz G. The diagnosis of pleural effusion by ultrasonic and radiologic techniques. Chest 1976;70:33-7

7 Feller-Kopman D. Ultrasound-guided thoracentesis. Chest 2006;129:1709-14

8 Barnes TW, Morgenthaler TI, Olson EJ, et al. Sonographically guided thoracentesis and rate of pneumothorax. J Clin Ultrasound 2005;33:442-6

9 Piccoli M, Trambaiolo P, Salustri A, et al. Bedside diagnosis and follow-up of patients with pleural effusion by a hand-carried ultrasound device early after cardiac surgery. Chest 2005;128:3413-20

10 Yang PC, Luh KT, Chang DB, et al. Ultrasonographic Evaluation of Pulmonary Consolidation. Am Rev Respir Dis 1992;146:757-62

11 Lichtenstein DA, Meziere GA, Lagoueyte JF, et al. A-lines and B-lines: lung ultrasound as a bedside tool for predicting pulmonary artery occlusion pressure in the critically ill. Chest 2009;136:1014-20

12 Ruskin JA, Gurney JW, Thorsen MK, et al. Detection of pleural effusions on supine chest radiographs. Am J Roentgenol 1987;148:681-3

13 Doust BD, Baum JK, Maklad NF, et al. Ultrasonic evaluation of pleural opacities. Radiology 1975;114:135-40

14 Remerand F, Dellamonica J, Mao Z, et al. Multiplane ultrasound approach to quantify pleural effusion at the bedside. Intensive Care Med 2010;36:656-64

15 McLoud TC, Flower CD. Imaging the pleura: sonography, CT, and MR imaging. Am J Roentgenol 1991;156:1145-53

16 Picano E. Sustainability of medical imaging. BMJ 2004;328:578-80

17 Xirouchaki N, Magkanas E, Vaporidi K, et al. Lung ultrasound in critically ill patients: comparison with bedside chest radiography. Intensive Care Med 2011;37:1488-93 